\title{
Importation of Human Seoul Virus Infection to Germany from Indonesia
}

\author{
Jörg Hofmann, ${ }^{1}$ Sabrina Weiss, ${ }^{1}$ \\ Martin Kuhns, Annekathrin Zinke, \\ Heike Heinsberger, Detlev H. Kruger
}

Seoul hantavirus-associated hemorrhagic fever with renal syndrome cases are rare outside Asia and have not yet been found in Germany. We report clinical and molecular evidence for a Seoul virus infection in a patient in Germany. The infection was most likely acquired during a stay in Sulawesi, Indonesia.

$\mathrm{H}_{2}$ antaviruses are globally emerging zoonotic pathogens that cause hemorrhagic fever with renal syndrome (HFRS) and hantavirus cardiopulmonary syndrome (1). One representative of this negative-sense, single-stranded RNA virus family is rat-associated Seoul virus (SEOV). In comparison to infections by prototypical Hantaan virus (HTNV), infection with SEOV is believed to lead to somewhat milder disease with shorter clinical phases (2). The most characteristic manifestations of SEOV infection are prominent abdominal symptoms, including hepatomegaly, hepatic dysfunction, and mild renal failure (2). A clear distinction from infections by related hantaviruses, such as HTNV, by serodiagnostic means is difficult and complicates interpretation of current clinical studies on (putatively) SEOVinfected patients.

SEOV infections have been found in rats and humans mainly in Asia but also worldwide (3). In Europe, molecular analysis has shown circulation of SEOV in wild brown rats (Rattus norvegicus) and in pet rats in the United Kingdom, France, Belgium, the Netherlands, and Sweden; however, human SEOV infections have been diagnosed by using only serologic analysis (4). Unequivocal molecular proof of human SEOV infections in Europe has been shown only for 4 patients in France (5) and 1 patient in the United Kingdom (https://www.gov.uk/government/publications/hantavirus-infection-in-people-sero-surveillancestudy-in-england).

Author affiliations: Charité-University of Medicine Institute of Virology, Berlin, Germany (J. Hofmann, S. Weiss, D.H. Kruger); Humboldt University, Berlin (J. Hofmann, S. Weiss, D.H. Kruger); Medilys Laborgesellschaft mbH, Hamburg, Germany (M. Kuhns); Asklepios Hospital Harburg, Hamburg (A. Zinke, H. Heinsberger)

DOI: https://doi.org/10.3201/eid2406.172044
In Germany, SEOV-specific antibodies or SEOV RNA have not been detected in rats or humans. We report on a case of molecularly proven hantavirus disease caused by SEOV infection in a patient in Germany who probably acquired the infection in Indonesia.

\section{The Patient}

On April 25, 2017, a 70-year-old man from Germany visited the emergency department at Asklepios Klinik Harburg (Hamburg, Germany) and reported severe diarrhea, thoracic/back pain, and bronchopulmonary symptoms. He also reported a fever that emerged at the end of a multiweek vacation on the island of Sulawesi in Indonesia a few days before his return to Germany on April 12. On May 2, he was hospitalized because of acute kidney injury.

We obtained laboratory findings for blood samples collected during the inpatient period of 11 days (Table). An initially low platelet count $(66 / \mathrm{nL})$ at admission returned to a reference value at day 8 of hospitalization. Serum creatinine levels were increased; maximum values were observed at days 2 and 3 of hospitalization. The glomerular filtration rate was decreased. Leukocyte counts and levels of C-reactive protein and lactate dehydrogenase were slightly increased. Increased levels of liver enzymes (aspartate aminotransferase, alanine aminotransferase, and $\gamma$-glutamyltransferase) indicated hepatic involvement, a characteristic of SEOV infections (2). Diuresis returned to reference values during hospitalization, and no polyuria was observed. The patient was discharged from the hospital with a serum creatinine level of $1.8 \mathrm{mg} / \mathrm{dL}$ and in largely normalized general condition.

We performed initial laboratory diagnostics (serologic analysis for hantavirus) by using the Hantavirus Profile 1 Immunoblot (Euroimmun, Lübeck, Germany) in the hospital laboratory and the recomLine HantaPlus IgG and IgM assays (Mikrogen, Martinsried, Germany) in our laboratory. The Profile 1 blot does not contain SEOV antigen, but serum from the patient showed reactivity for DobravaBelgrade virus (DOBV) IgG and HTNV IgM. The recomLine IgG and IgM blots showed reactivity for DOBV, HTNV, and SEOV; the weakest result was for SEOV nucleocapsid protein.

For molecular typing, we tested the first blood sample collected during hospitalization by using reverse transcription for hantavirus RNA. We obtained partial

${ }^{1}$ These authors contributed equally to this article. 
Table. Biochemical parameters of a case-patient during 11 days of hospitalization who was infected with Seoul virus imported to Germany from Indonesia*

\begin{tabular}{|c|c|c|c|c|c|c|}
\hline Parameter & Reference range & Day 1 & Day 2 & Day 3 & Day 9 & Day 11 \\
\hline Platelet count & 160-370/nL & 66 & 85 & 112 & 394 & 490 \\
\hline Creatinine & $0.7-1.2 \mathrm{mg} / \mathrm{dL}$ & 3.7 & 5.5 & 5 & 2.3 & 1.8 \\
\hline GFR CKD-EPI & $>60 \mathrm{~mL} / \mathrm{min}$ & 16 & 10 & 11 & 28 & 37 \\
\hline Leukocyte count & $3.5-9.8$ cells/nL & 12.8 & 15.7 & 16.8 & 12.1 & 11.7 \\
\hline CRP & $<5.0 \mathrm{mg} / \mathrm{L}$ & 54.4 & ND & 26.5 & 10.4 & ND \\
\hline LDH & $<250 \mathrm{U} / \mathrm{L}$ & 735 & 561 & ND & 285 & ND \\
\hline GGT & $<60 \mathrm{U} / \mathrm{L}$ & 137 & 115 & 121 & 159 & ND \\
\hline ALT & $<50 \mathrm{U} / \mathrm{L}$ & 75 & 119 & 234 & 120 & ND \\
\hline AST & $<50 \mathrm{U} / \mathrm{L}$ & ND & 173 & ND & 46 & ND \\
\hline Urea & $18-55 \mathrm{mg} / \mathrm{dL}$ & ND & 240 & ND & 73 & 48 \\
\hline Hemoglobin & $13.5-17.5 \mathrm{~g} / \mathrm{dL}$ & 17.6 & 16 & 15.1 & 12.9 & 11.4 \\
\hline
\end{tabular}

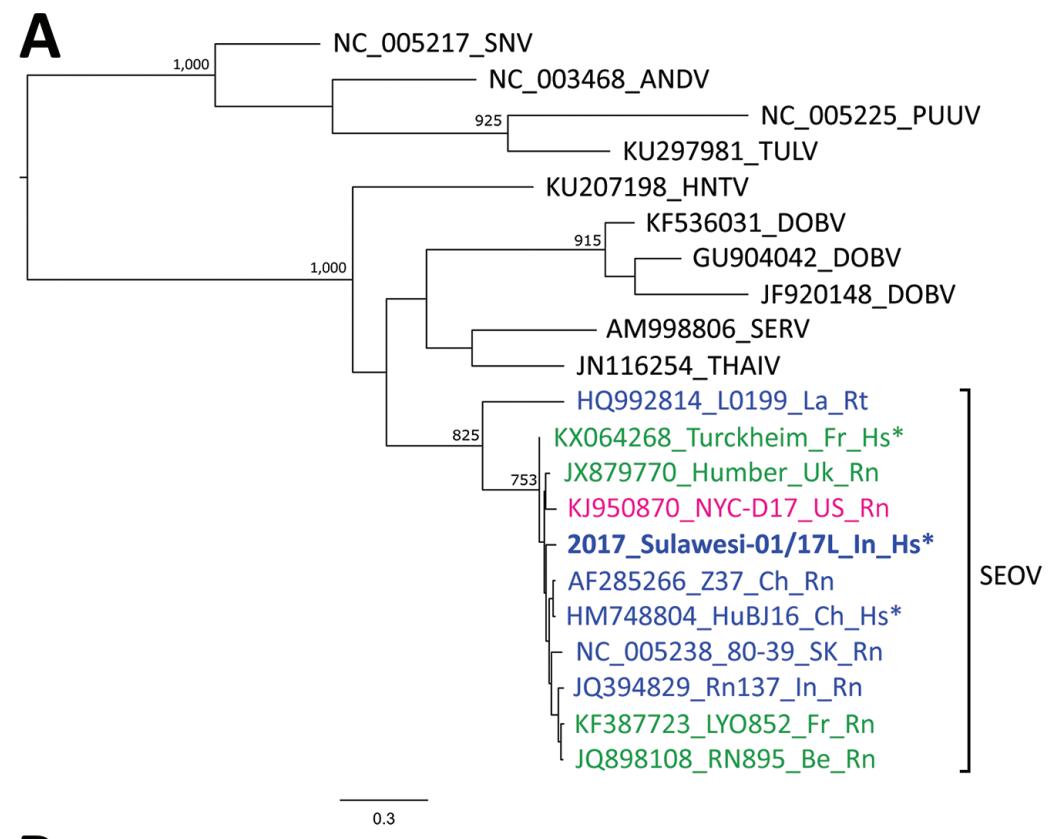

B

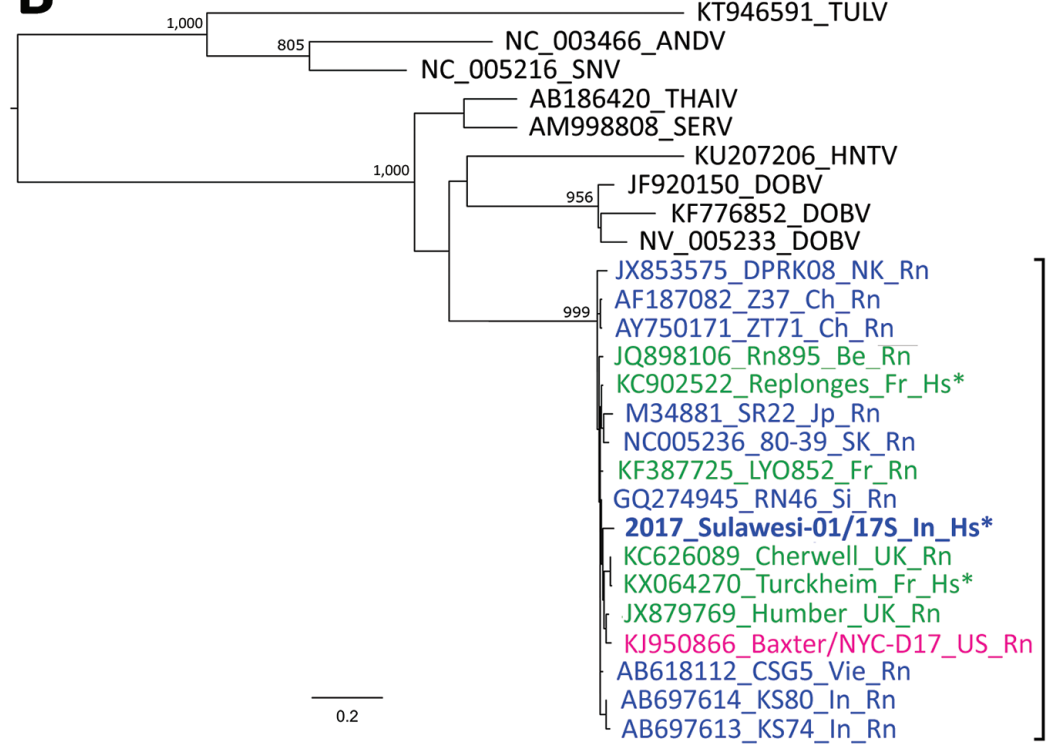

Figure. Maximum-likelihood phylogenetic trees of partial RNA segments of orthohantaviruses. A) Large RNA segments based on a 347-nt alignment and the general time reversible plus gamma distribution model of nucleotide substitution. B) Small RNA segments based on a 318-nt alignment and the Hasegawa-Kishino-Yano 85 plus gamma distribution model. Trees were constructed by using PhyML3.0 (8) and the best-fitting model according to smart model selection in this software and 1,000 bootstrap replicates. Values along branches are bootstrap values $>75 \%$ for major clades. GenBank accession number, strain, country of origin, and host are shown for each virus isolate. Bold indicates SEOV isolated from the patient in this study. Blue indicates SEOV strains from Asia, green indicates SEOV strains from Europe, and red indicates SEOV strains from the Americas. Scale bars indicate nucleotide substitutions per site. *Sequences from viruses of human origin. ANDV, Andes virus; Be, Belgium; Ch, China; DOBV, DobravaBelgrade virus; Fr, France; HNTV, Hantaan virus; Hs, Homo sapiens; In, Indonesia; Jp, Japan; La, Laos; NK, North Korea; PUUV, Puumala virus; Rn, Rattus norvegicus; Rt, $R$. tanezumi; SEOV, Seoul virus; SERV, Serang virus; Si, Singapore; SK, South Korea; SNV, Sin nombre virus; THAIV, Thailand virus; TULV, Tula virus; UK, United Kingdom; US, United States; 
sequences of genes coding for hantavirus polymerase (large [L] RNA segment) by using HAN-L primers (6). We also obtained nucleocapsid protein (small [S] RNA segment) sequences by using primers $\mathrm{S} 1$ and $\mathrm{S} 2$ (7) and primers S598 (5'-ATG AAG GCA GAA GAG ATT ACA CC[TA] GG-3') and S6HC (5'-CCA GCA AAC ACC CAT ATT GAT GAT-3') as nested primers. Sequences of the strain Sulawesi-01/17 were deposited in GenBank under accession nos. MG386252 for the L segment and MG386253 for the S segment. We showed by phylogenetic analysis that $\mathrm{L}$ and $\mathrm{S}$ sequences obtained from the patient segregated to the main cluster of SEOV strains, which clearly demonstrated that SEOV was the causal agent of infection (Figure).

\section{Conclusions}

We report an infection with SEOV in a patient in Germany. If one considers the clinical course, this case of HFRS appeared moderate, and the outcome for this casepatient was favorable and showed a full recovery. As expected for SEOV-associated hantavirus disease (2), severe gastrointestinal symptoms and liver involvement were observed, but kidney dysfunction was mild and no hemodialysis was needed. However, the clinical course appeared unusually protracted $(\approx 6$ weeks) between the febrile phase and discharge from hospital. Typing of the causative hantavirus by using 2 commercial immunoblots was misleading, but sequence data obtained from the $\mathrm{L}$ and $\mathrm{S}$ segments unequivocally confirmed that the patient was infected with SEOV.

Molecular phylogenetic analysis of genetically characterized SEOV strains resulted in creation of 4 phylogroups (9). Most strains, including all strains from locations other than mountainous areas of China, belong to major phylogroup A and have probably spread from Asia as a result of distribution of rats during trade activities by humans (9). Although SEOV strains are found in ports and countries with overseas traffic, no SEOV infections of rats or humans have been reported in Sulawesi in Indonesia. SEOV RNA in rats, but no proof of human infections, was reported in Jakarta, on the island of Java in Indonesia $(10,11)$.

Because of the wide spread of SEOV caused by extensive movement of its natural host (rats), analysis of the nucleotide sequence of the SEOV strain from our patient does not enable identification of the place of infection. This strain is related to SEOV strains from Asia, Europe, and the United States (Figure). This finding contrasts with those for other hantaviruses, for which there are clear spatial association between virus strains and carrier hosts, such as vole-associated Puumala virus $(6,12)$.

Although molecular characterization of the SEOV strain isolated from the patient enabled diagnosis of SEOV infection, these results did not identify the original place of infection. The clinical course, including onset of disease, makes ithighly probable that the patientacquired the infection during his stay in Indonesia. Because he did not report any further travel activities during this stay, we conclude that he had acquired the infection in Sulawesi in Indonesia.

In summary, we report a human SEOV infection imported to Germany from Indonesia. Our results demonstrate that extended molecular diagnostics are required for reliable hantavirus typing, especially for patients with travel histories.

\section{Acknowledgments}

We thank B. Klempa and M. Raftery for critically reading the manuscript and for helpful discussions, R. Neitz for providing information about the case to the National Consultation Laboratory and contacting the case-patient, and B. Auste and C. Stephan for excellent technical assistance.

This study was supported by the Robert Koch Institute with funds from the Federal Ministry of Health in Germany (grant no. 1369-382) and the Federal Ministry of Education and Research (grant no. 01KI1721C). S.W. was supported by the Federal Ministry of Education and Research (grant no. 031L0096B).

\section{About the Author}

Dr. Hofmann is a clinical virologist at the National Consultation Laboratory for Hantaviruses, Institute of Virology, Charité University of Medicine, Berlin, Germany. His primary research interest is human infections with viral pathogens.

\section{References}

1. Kruger DH, Figueiredo LT, Song JW, Klempa B. Hantaviruses: globally emerging pathogens. J Clin Virol. 2015;64:128-36. http://dx.doi.org/10.1016/j.jcv.2014.08.033

2. Lee HW. Hemorrhagic fever with renal syndrome in Korea. Rev Infect Dis. 1989;11(Suppl 4):S864-76. http://dx.doi.org/10.1093/ clinids/11.Supplement_4.S864

3. Clement J, Maes P, Van Ranst M. Hemorrhagic fever with renal syndrome in the new, and hantavirus pulmonary syndrome in the Old World: paradi(se)gm lost or regained? Virus Res. 2014; 187:55-8. http://dx.doi.org/10.1016/j.virusres.2013.12.036

4. Goeijenbier M, Verner-Carlsson J, van Gorp EC, Rockx B, Koopmans MP, Lundkvist $\AA$, et al. Seoul hantavirus in brown rats in the Netherlands: implications for physicians - epidemiology, clinical aspects, treatment and diagnostics. Neth J Med. 2015; 73:155-60.

5. Reynes JM, Carli D, Bour JB, Boudjeltia S, Dewilde A, Gerbier G, et al. Seoul virus infection in humans, France, 20142016. Emerg Infect Dis. 2017;23:973-7. http://dx.doi.org/10.3201/ eid2306.160927

6. Klempa B, Fichet-Calvet E, Lecompte E, Auste B, Aniskin V, Meisel H, et al. Hantavirus in African wood mouse, Guinea. Emerg Infect Dis. 2006;12:838-40. https://doi.org/10.3201/ eid1205.051487

7. Sibold C, Sparr S, Schulz A, Labuda M, Kozuch O, Lysý J, et al. Genetic characterization of a new hantavirus detected in Microtus arvalis from Slovakia. Virus Genes. 1995;10:277-81. http://dx.doi.org/10.1007/BF01701817 
8. Guindon S, Dufayard JF, Lefort V, Anisimova M, Hordijk W, Gascuel O. New algorithms and methods to estimate maximumlikelihood phylogenies: assessing the performance of PhyML 3.0. Syst Biol. 2010;59:307-21. http://dx.doi.org/10.1093/sysbio/ syq010

9. Lin XD, Guo WP, Wang W, Zou Y, Hao ZY, Zhou DJ, et al. Migration of Norway rats resulted in the worldwide distribution of Seoul hantavirus today. J Virol. 2012;86:972-81. http://dx.doi.org/10.1128/JVI.00725-11

10. Plyusnina A, Ibrahim IN, Winoto I, Porter KR, Gotama IB, Lundkvist A, et al. Identification of Seoul hantavirus in Rattus norvegicus in Indonesia. Scand J Infect Dis. 2004;36:356-9. http://dx.doi.org/10.1080/00365540410019264
11. Ibrahim IN, Shimizu K, Yoshimatsu K, Yunianto A, Salwati E, Yasuda SP, et al. Epidemiology of hantavirus infection in Thousand Islands regency of Jakarta, Indonesia.J Vet Med Sci. 2013;75:1003-8. http://dx.doi.org/10.1292/ jvms.12-0442

12. Ettinger J, Hofmann J, Enders M, Tewald F, Oehme RM, Rosenfeld UM, et al. Multiple synchronous outbreaks of Puumala virus, Germany, 2010. Emerg Infect Dis. 2012; 18:1461-4. http://dx.doi.org/10.3201/eid1809.111447

Address for correspondence: Jörg Hofmann, Institute of Virology, Helmut-Ruska-Haus, Charité University of Medicine, Berlin, Germany; email: joerg.hofmann@charite.de
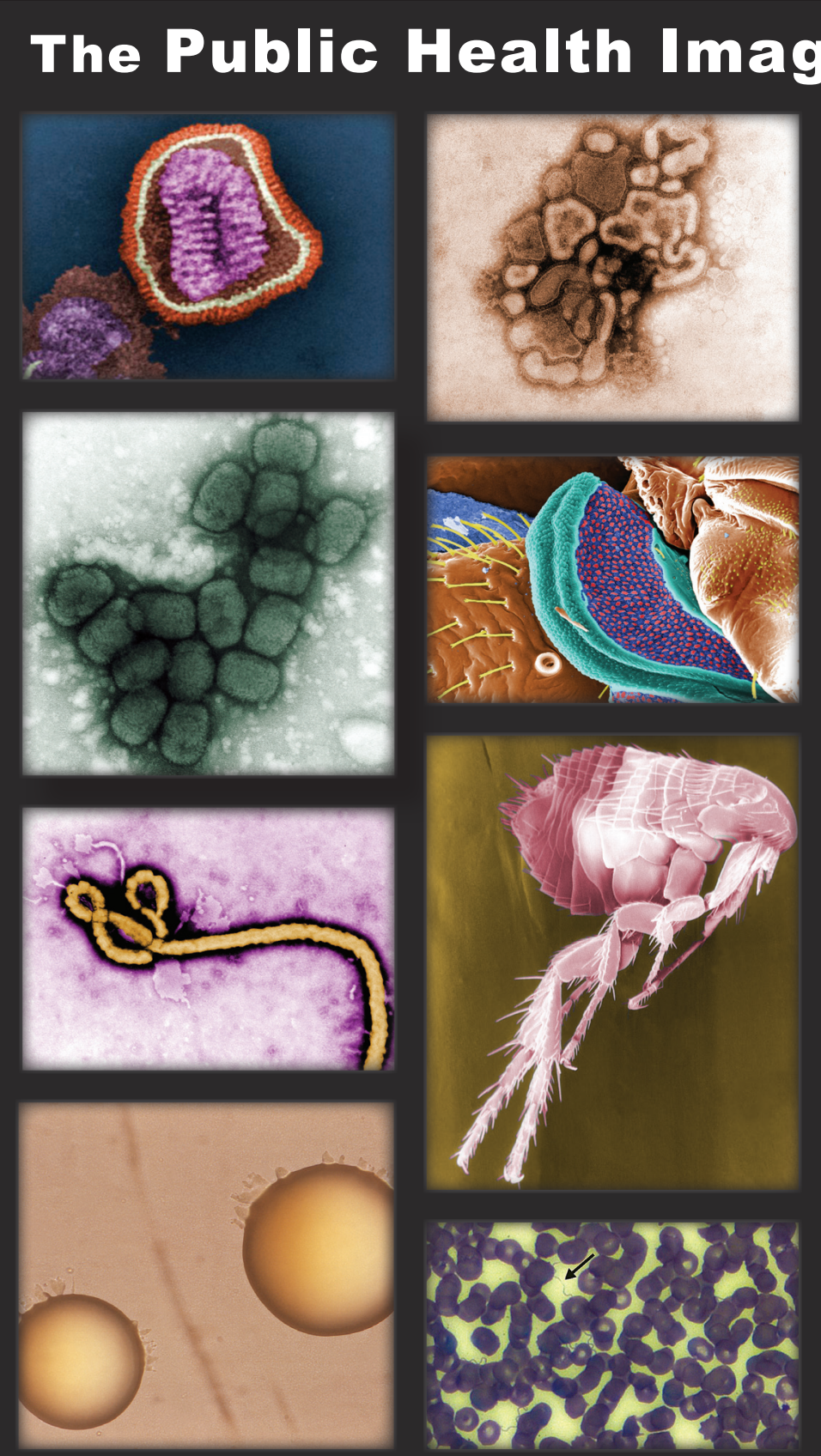

The Public Health Image Library (PHIL), Centers for Disease Control and Prevention, contains thousands of public healthrelated images, including high-resolution (print quality) photographs, illustrations, and videos.

PHIL collections illustrate current events and articles, supply visual content for health promotion brochures, document the effects of disease, and enhance instructional media.

PHIL images, accessible to PC and Macintosh users, are in the public domain and available without charge.

Visit PHIL at: http:/ /phil.cdc.gov/phil 